Théologiques

Revue interdisciplinaire d'études religieuses

Théologiques

\title{
La méditation de pleine conscience dans l'intervention en soins spirituels avec les patients en psychiatrie
}

\author{
Bruno Bélanger, Cécile Charbonneau, Line Beauregard, Christiane Biron et \\ Stéphane Poulin
}

Volume 28, numéro 1, 2020

URI : https://id.erudit.org/iderudit/1074681ar

DOI : https://doi.org/10.7202/1074681ar

Aller au sommaire du numéro

Éditeur(s)

Institut d’études religieuses de l’Université de Montréal

ISSN

1188-7109 (imprimé)

1492-1413 (numérique)

Découvrir la revue

Citer cet article

Bélanger, B., Charbonneau, C., Beauregard, L., Biron, C. \& Poulin, S. (2020). La méditation de pleine conscience dans l'intervention en soins spirituels avec les patients en psychiatrie. Théologiques, 28(1), 197-221.

https://doi.org/10.7202/1074681ar

\section{Résumé de l'article}

$\mathrm{Au}$ cours des dernières années, dans la foulée du programme de méditation pleine conscience élaboré par Jon Kabat Zinn, les services de soins spirituels intègrent de plus en plus la médiation au sein de leurs interventions. Cet article présente les résultats d'une recherche visant à évaluer les effets d'un programme de méditation "Soin spirituel pour un mieux-être » sur diverses dimensions psychiques et spirituelles. Les données ont été recueillies auprès de 21 patients suivis en externe en psychiatrie à l'Hôpital de l'Enfant-Jésus selon 3 temps de mesure : avant le programme, immédiatement à la fin du programme et 3 mois après. Les résultats suggèrent une amélioration chez les patients immédiatement à la fin du programme mais qui tend à s'atténuer 3 mois plus tard. Cette recherche pose, entre autres, des questions concernant les mesures d'évaluation de la dimension spirituelle. 


\title{
La méditation de pleine conscience dans l'intervention en soins spirituels avec les patients en psychiatrie
}

\author{
Bruno Bélanger \\ Cécile Charbonneau \\ Line Beauregard \\ Agent et agentes de planification, \\ programmation et recherche
}

Centre Spiritualitésanté de la Capitale-Nationale

Christiane BIRON

Intervenante en soins spirituels

Centre Spiritualitésanté de la Capitale-Nationale

Stéphane Poulin

Psychiatre

Hôpital de l'Enfant-Jésus"

Depuis une vingtaine d'années, les questions entourant l'intégration de la dimension spirituelle dans les soins aux patients ont fait l'objet de nombreuses publications scientifiques (Fitchett et Canada 2010; Koenig 2004 ; 2012 ; Murray et al. 2004 ; Okon 2005 ; Puchalski 2007 ; Sinclair et al. 2006 ; Visser et al. 2010). Au sein de cette importante littérature, on retrouve un sous-ensemble touchant les effets de la pratique de la méditation, particulièrement de pleine conscience, sur la santé physique et mentale. Ce sous-ensemble comportant de nombreuses études issues du champ de la psychologie et de la psychiatrie cherche, notamment, à évaluer le processus par lequel la méditation produit des effets positifs (Baer 2003 ; Brown et al. 2007 ; Grabovac 2014 ; Shapiro et al. 2006). Cependant, au

Exceptionnellement, on trouvera la notice de présentation des auteurs et autrices à la fin de l'article.

(C) Revue Théologiques 2020. Tout droit réservé. 
sein de ce dernier corpus, peu de travaux de recherche proviennent du champ de l'intervention spirituelle. De plus, peu d'études ont exploré les liens entre la pratique de la méditation et la dimension spirituelle des patients. Cet article présente les résultats d'une recherche réalisée par le Centre Spiritualitésanté de la Capitale-Nationale (ci-après CSsanté) ${ }^{1}$. Celleci visait à évaluer les effets d'un programme de méditation élaboré par le CSsanté, essentiellement inspiré des ateliers de pleine conscience développés par Jon Kabat-Zinn (1982), sur les dimensions psychique et spirituelle des patients.

\section{La méditation de pleine conscience et ses effets sur le psychisme}

La méditation de pleine conscience (MPC) (en anglais mindfulness; KabatZinn 1982) s'enracine dans les différents courants de la pratique méditative bouddhiste (Kabat-Zinn 2003). Développée par Kabat-Zinn $(1982)^{2}$ et se voulant dénuée de toute connotation religieuse, cette pratique est à la source d'un immense champ de recherche. La MPC se caractérise par la conscience du moment présent dans le non-jugement. Plutôt que de restreindre l'attention sur un objet (comme dans la méditation de concentration), l'accent est placé sur l'observation, par le méditant, de tous les phénomènes qui entrent dans son champ de conscience. Ainsi, les sensations physiques, les perceptions, les cognitions, les émotions sont observées soigneusement, mais sans être évaluées en tant que phénomènes bons ou mauvais, justes ou faux, sains ou malsains ou encore importants ou insignifiants. La pleine conscience se définit donc comme étant l'observation, sans jugement, du flot continu des stimuli internes et externes tels qu'ils se déploient.

1 Le Centre Spiritualitésanté de la Capitale-Nationale (CSsanté) assure le fonctionnement au quotidien des services de soins spirituels répartis dans plus de 30 sites du réseau de la santé et des services sociaux de la ville de Québec et de ses environs. Il est constitué d'une quarantaine d'intervenants et d'intervenantes en soins spirituels. Ce projet de recherche a été mené par une équipe interdisciplinaire (les signataires de cet article).

2 En 1982, Jon Kabat-Zinn élabore le programme MBSR (Mindfulness-Based Stress Reduction) visant la réduction du stress. Son programme, développé dans un cadre de médecine comportementale, avait pour but d'aider les patients à faire face au stress, à la douleur chronique et à la maladie grâce à la MPC. 
De nombreuses études tendent à établir des liens entre la pratique de la méditation et l'amélioration de la santé mentale et physique des patients (Baer 2003 ; Carlson et al. 2001 ; Chen et al. 2012 ; Creswell et Lindsay 2014 ; Evans et al. 2008 ; Gonçalves et al. 2015 ; Hofmann et al. 2010 ; Speca et al. 2000). Par exemple, Heeren et Philippot (2011) ont constaté une diminution de psychopathologies à la suite d'un entrainement à la pleine conscience. Ils ont, entre autres, observé une réduction des pensées ruminantes inadaptées (repenser sans cesse à quelque chose) et une augmentation de pensées adaptatives chez les personnes anxieuses. Barnhofer et al. (2009) ainsi qu'Evans et al. (2008) ont noté une réduction significative des symptômes de dépression chez les personnes ayant participé à une thérapie cognitive de groupe basée sur la pleine conscience comparativement à ceux qui avaient reçu uniquement le traitement habituel. L'efficacité de la MPC a fait l'objet de nombreuses revues systématiques et méta-analyses. Ces dernières concluaient que cette forme de méditation est une approche prometteuse (Bawa et al. 2015 ; Khoury et al. 2013 ; Strauss et al. 2014 ; Zhang et al. 2015). Cependant, les conclusions en regard de l'efficacité des thérapies de pleine conscience varient. Les résultats de l'étude de Bawa $e t$ al. (2015) démontrent que la MPC a une efficacité limitée en ce qui a trait à la gestion de la douleur. La méta-analyse effectuée par Strauss et al. (2014) suggère que les personnes ayant un épisode actif de dépression peuvent bénéficier d'approches de pleine conscience, mais cette conclusion ne s'applique pas de manière aussi saillante dans le cas des personnes éprouvant de l'anxiété, ce qui contraste avec d'autres méta-analyses ayant démontré l'efficacité de la MPC pour réduire l'anxiété (Khoury et al. 2013 ; Zhang et al. 2015). Plusieurs facteurs peuvent expliquer l'inconsistance des résultats concernant l'efficacité des thérapies de pleine conscience : 1) la forme de thérapie (Chan et Larson 2015 ; Zhang et al. 2015 ; Khoury et al. 2013) ; 2) le type de problème de santé étudié (Chan et Larson 2015) ; 3) la présence ou l'absence d'un suivi à long terme (Zhang et al. 2015); 4) le devis de recherche utilisé (Marchand 2013); et 5) les différents médiateurs impliqués tels que la durée du traitement, les pratiques à la maison, la régularité de la présence aux ateliers et la formation et l'expérience de l'enseignant (Khoury et al. 2013). Ainsi, malgré les résultats généralement positifs et prometteurs, de nombreux auteurs concluent que plus de recherches doivent être réalisées afin de mieux comprendre les impacts de la pratique de la méditation et ses mécanismes d'action (Bawa et al. 2105 ; Fournier 2014 ; Jain et al. 2015 ; Zhang et al. 2015). 


\section{Les effets de la méditation de pleine conscience sur la dimension spirituelle}

Même si la méditation a été adaptée à des fins profanes, certains auteurs (Baer 2010 ; Carmody et al. 2008 ; Greeson et al. 2011 ; 2015) ont étudié les liens avec la dimension spirituelle des patients. Selon Carmody et al. (2008), la dimension spirituelle est considérée par de nombreux chercheurs comme un aspect fondamental de la personne constituant un élément significatif de sa qualité de vie et de son bien-être global. Ces auteurs ont cherché à mieux cerner l'impact ou les répercussions des ateliers de pleine conscience MBSR (Mindfulness-Based Stress Reduction) sur divers aspects de la vie des participants, dont la spiritualité évaluée l'aide du FACITSP (Functional Assessment of Chronic Illness Therapy-Spiritual well-being scale), considéré comme l'outil le plus adéquat pour examiner cette dimension dans un contexte séculier. En plus de réduire les symptômes de détresse psychologique et les symptômes médicaux déclarés, la participation au programme MBSR augmente la pleine conscience et le bien-être spirituel. L'étude montre également une association entre la pleine conscience et le bien-être spirituel, notamment lorsqu'il est question de sens et de paix intérieure. Cependant, certaines questions subsistent en lien avec ces résultats positifs, notamment quant à l'influence de la vie spirituelle des participants avant le programme (leurs croyances, leurs expériences, leurs pratiques) (Greeson et al. 2015) et au choix du ou des concepts mesurés par l'outil retenu dans le protocole de la recherche (Carmody et al. 2008).

\section{Intervention}

Jusqu'à tout récemment dans l'ensemble de leurs pratiques d'intervention, il était peu fréquent que les intervenants en soins spirituels (ISS) du CSsanté s'inspirent des outils de la méditation dans leur travail d'accompagnement. C'est en 2014 qu'une expérience pionnière dans le champ spécifique du soin spirituel a été proposée à la clinique externe de psychiatrie de l'Hôpital de l'Enfant-Jésus : un programme de méditation de huit semaines a été offert aux patients aux prises avec des problématiques de dépression ou d'anxiété. Ce programme, qui porte le nom de Soin spirituel pour un mieux-être, a été élaboré en s'inspirant du contenu des ateliers de MBSR de Kabat-Zinn par une ISS du CSsanté et coauteure de cet article (Christiane 
Biron). Le programme MBSR se veut exempt de toute connotation religieuse et il ne propose pas de relation avec un Transcendant. Toutefois, étant donné que nous œuvrons dans un contexte d'intervention en soins spirituels, nous souhaitions inclure dans le programme des éléments d'ouverture à une Transcendance. L'élément distinct le plus important du programme original de Kabat-Zinn inclus dans notre programme donnait aux participants la possibilité de faire référence à leur propre conception de la Transcendance (Biron 2014).

Dans le cadre de cette recherche, nous avons vérifié les effets de ce programme sur deux grandes dimensions : 1 ) psychique, afin de vérifier si les effets du programme sont similaires à ceux retrouvés dans la littérature et 2) spirituelle, afin d'évaluer l'impact de l'introduction de celle-ci dans le programme. Le contexte dans lequel nous évoluons et le sens de nos interventions nous ont menés à porter une attention particulière à cette deuxième dimension.

Nous avons donc cherché à évaluer les effets de ce programme sur des patients à la suite d'une hospitalisation en psychiatrie. Nous souhaitions vérifier si ce programme permettait aux participants : 1) d'acquérir les principes de base de la méditation (dont, notamment, l'augmentation de la présence attentive) ;2) de réduire les symptômes d'humeur dépressive et d'anxiété ; 3) de diminuer les pensées répétitives non constructives et d'augmenter les pensées répétitives constructives ; 4) d'augmenter la compassion envers eux-mêmes ; 5) de modifier le bien-être spirituel ; et 6) de modifier l'expérience spirituelle. Nous émettons donc l'hypothèse qu'un exercice de méditation intégré à la vie quotidienne peut servir de soutien et devenir un outil de prévention des rechutes dans le traitement de la dépression et de l'anxiété et qu'il peut avoir des effets bénéfiques sur la spiritualité des participants.

\section{Méthodologie}

\subsection{Les participants}

Pour participer au programme Soin spirituel pour un mieux-être, la personne devait bénéficier d'un suivi en psychiatrie externe à l'Hôpital de l'EnfantJésus. Williams et al. (2009) suggèrent d'attendre que ces personnes soient 
sorties de la phase aiguë de la maladie avant d'entreprendre ce type de travail. Une fois informée du programme par leur psychiatre traitant, la personne intéressée communiquait directement avec la responsable des ateliers. Les personnes admissibles devaient rencontrer les critères d'inclusion suivants : 1) avoir un diagnostic d'épisode dépressif majeur, épisode dépressif caractérisé ou de trouble anxieux (d'après le DSM IV ou le DSM V, selon le moment où le diagnostic a été posé) ; 2) bénéficier d'un suivi externe par un psychiatre de l'Hôpital de l'Enfant-Jésus ; 3) avoir 18 ans ou plus ; et 4) parler français. Étaient exclues les personnes qui avaient des idées suicidaires, une dépendance (drogue, alcool) ou des symptômes psychotiques.

\subsection{Procédure}

Les personnes intéressées à participer à l'évaluation du programme de méditation ont été rencontrées en groupe une heure avant le début du programme de méditation (Temps 1) par une personne autre que la responsable des ateliers. Cette rencontre visait d'abord à obtenir leur consentement éclairé ${ }^{3}$ et ensuite, à compléter un bref questionnaire de renseignements généraux ainsi que tous les instruments de mesure permettant de vérifier les effets du programme sur les variables retenues (Temps 1 ou T1). À la fin du programme de huit semaines, les participants devaient compléter de nouveau les mêmes instruments de mesure à deux reprises : immédiatement après la fin du programme (Temps 2 ou T2) et trois mois plus tard (Temps 3 ou T3). Au total, 21 personnes ont participé à la recherche, réparties en 4 groupes.

\subsection{Description du programme de méditation » Soin spirituel pour un mieux- être»}

Les ateliers de méditation ont été tenus pendant huit semaines consécutives à raison d'une rencontre hebdomadaire d'une durée de 90 minutes. Lors du premier atelier, la personne responsable informe les participants que différents textes réflexifs de nature philosophique et spirituelle leur seront proposés lors des rencontres. Elle signale également qu'ils sont libres, tout

3 Ce projet a reçu l'approbation éthique du comité d'éthique de la recherche du CHU de Québec. 
au long de la démarche, de partager leurs propres croyances en lien avec leur foi. Comme présenté au tableau 1 , chaque semaine porte sur un thème spécifique.

Tableau 1 - Thèmes des rencontres hebdomadaires

\begin{tabular}{|c|l|}
\hline Semaine & \multicolumn{1}{c|}{ Thèmes } \\
\hline 1 & Présence à soi par la respiration \\
\hline 2 & Conscience du corps dans sa globalité \\
\hline 3 & Donner du poids aux expériences agréables \\
\hline 4 & Répondre avec compétence et grâce aux expériences désagréables \\
\hline 5 & $\begin{array}{l}\text { Conscientiser l'influence de nos pensées négatives et sortir de leur } \\
\text { emprise }\end{array}$ \\
\hline 6 & Cultiver la bienveillance \\
\hline 7 & Contacter ce qui est stable au plus profond de soi \\
\hline 8 & Habiter sa vie \\
\hline
\end{tabular}

\subsection{Déroulement des rencontres}

Chaque rencontre se déroule selon la séquence suivante : accueil ( $5 \mathrm{mi}-$ nutes) ; retour sur la semaine (20 minutes); présentation du thème de la rencontre (15 minutes); expérience de méditation (30 minutes); et retour sur cette expérience ( 20 minutes).

\subsection{Instruments de mesure}

Les outils de mesure retenus ont déjà été utilisés dans diverses études ayant pour but d'évaluer les effets de la pratique de la MPC sur le psychisme. Ils visent à vérifier si les objectifs du programme de méditation Soin spirituel pour un mieux-être ont été atteints par les participants.

L'échelle de mesure FMI-14 (Freiburg Mindfulness Inventory14 items; Walach et al. 2006) est une forme abrégée du FMI qui permet de vérifier si les participants ont intégré les principes de base de la méditation (objectif 1). Cette échelle, fiable et cohérente, évalue plusieurs aspects de la pleine conscience comme l'observation sans jugement du moment présent ainsi que l'ouverture à l'expérience négative. Chaque item est évalué en utilisant une échelle de type Likert en quatre points allant de 1 (presque jamais) à 4 (presque toujours). Le score peut être considéré globalement ou 
être décomposé sur le plan de l'observation sans jugement que la personne fait du moment présent (FMIP) ainsi que son ouverture à l'expérience négative, soit son acceptation (FMIA). La version française de l'échelle FMI-14 offre une cohérence interne moyenne ( $\alpha=0,74)$ (Trousselard et al. 2010).

Le POMS-SF (Profile of Mood States - Short Form) est un instrument de mesure global des états d'humeur et de leurs fluctuations et évalue si le programme permet de diminuer l'humeur dépressive et l'anxiété (objectif 2). Le POMS-SF (Shacham 1983) est la forme abrégée du POMS développé par McNair et al. (1971) sur la base d'une série d'analyses factorielles desquelles six facteurs ont été dégagés : 1) Vigueur/Activité, 2) Colère/Hostilité, 3) Tension/Anxiété, 4) Dépression/Déception, 5) Incertitude/Confusion et 6) Fatigue/Manque d'énergie. Dans la formule abrégée, ces différents facteurs reposent sur 37 adjectifs, cotés sur un continuum en cinq points allant de 0 (pas du tout) à 4 (extrêmement), et chacun affiche de bons indices de coefficient interne (alphas allant de 0,80 à 0,91). Le POMSSF a été validé par Shacham (1983) puis adapté en français par Fillion et Gagnon (1999). On peut calculer un score pour chacun des facteurs ou encore on peut calculer un score global comme indice d'évaluation approfondie de l'humeur, lorsque l'ensemble des items sont considérés.

Le Mini-CERTS (Mini-Cambridge Exeter Repetitive Thought Scale) évalue la présence de pensées ruminantes (objectif 3) (Heeren et Philippot 2011). Celles-ci peuvent être examinées en tenant compte du score global, mais aussi en distinguant les pensées ruminantes adaptatives (RA) et les pensées ruminantes inadaptées (RI). Chacun des 15 items sont observés à l'aide d'une échelle de type Likert en quatre points allant de 1 (presque jamais) à 4 (presque toujours). La version française (Douilliez et Philippot 2012) indique l'existence d'une cohérence interne moyenne pour chacune de ces sous-échelles (RA: $\alpha=0,77 ; \mathrm{RI}: \alpha=0,75$ ).

Le SCS-SF, version française du Self-Compassion Scale, est une échelle de 12 items développée par Neff (2003) qui vise à mesurer, dans des situations d'échec, à quel point une personne fait montre de compassion ou d'autocritique envers elle-même (objectif 4). Les aspects évalués portent sur la perception de ses expériences : les expériences sont-elles perçues comme faisant partie de l'expérience humaine au sens large ou sont-elles envisagées comme étant isolées? La personne regarde-t-elle les pensées et les sentiments douloureux en pleine conscience ou s'identifie-t-elle à eux ? Cette 
échelle est reliée à une réduction des symptômes de dépression et d'anxiété et à une plus grande satisfaction en regard de sa vie (Neff 2003).

Le FACIT-SP (The Functional Assessment of Chronic Illness TherapySpiritual well-being scale) est un questionnaire composé de 12 items développés par Peterman et al. (2002) pour mesurer le bien-être spirituel de personnes souffrant de dépression ou d'anxiété à la suite d'un diagnostic de cancer ou d'une autre maladie chronique. Des patients, des psychothérapeutes et des experts religieux/spirituels ont participé à l'élaboration des items en décrivant les aspects de la spiritualité ou de la foi qui contribuaient selon eux à la qualité de vie. Les principaux aspects qui ressortent de cet exercice sont le sens donné à la vie, les sentiments de paix et d'harmonie ainsi que les sentiments de force et de réconfort découlant de sa spiritualité. Chaque item est coté sur un continuum en cinq points allant de 0 (pas du tout) à 4 (extrêmement). La FACIT-SP offre une bonne cohérence interne $(\alpha=0,87)$. Cet instrument de mesure a été utilisé dans des dizaines d'études examinant les relations entre le bien-être spirituel, la santé et l'ajustement à la maladie. Peterman et al. (2002) ont souligné une corrélation négative intéressante de cette échelle avec le score global du POMS. Son utilisation dans la présente recherche permettra de mesurer si le programme modifie le bien-être spirituel des participants (objectif 5).

Le DSES (Daily Spiritual Experience Scale) est un instrument d'autoévaluation conçu par Underwood (2006) mesurant comment l'expérience spirituelle s'exprime dans la vie quotidienne des gens. L'échelle de 16 items mesure des éléments tels que la crainte, la gratitude, la miséricorde, le lien avec le Transcendant, la compassion et le désir de proximité avec Dieu, le divin ou le sacré (ex. : essence divine, esprit divin, pouvoir suprême). Cet instrument a été développé initialement pour des études en santé et il est fréquemment utilisé (Underwood 2019) dans le cadre d'évaluation de programme ainsi que pour examiner tout changement dans les expériences religieuses ou spirituelles au fil du temps (objectif 6).

\subsection{Analyses}

Afin de déterminer les bénéfices du programme, des tests T pairés ont été effectués. Pour chacune des variables mesurées, la différence entre T1 et T2 a été vérifiée, de même que celle entre T1 et T3. 


\section{Résultats}

Comme indiqué précédemment, 21 personnes ont participé au projet de recherche. Parmi elles, 13 ont complété le programme et les questionnaires (T2), et 9 ont été réévaluées 3 mois après la fin du programme (T3). Le tableau 2 présente les caractéristiques sociodémographiques des 21 participants qui ont répondu aux questionnaires.

Tableau 2 - Données sociodémographiques des participants

\begin{tabular}{|c|c|c|}
\hline \multicolumn{2}{|c|}{ Caractéristiques } & $N=21$ \\
\hline \multirow[t]{2}{*}{ Sexe : } & Féminin & 14 \\
\hline & Masculin & \\
\hline \multirow[t]{3}{*}{ Nbr d'enfants : } & 0 & 9 \\
\hline & 1 & 5 \\
\hline & 2 et + & 7 \\
\hline \multirow[t]{3}{*}{ Revenus : } & - de $15000 \$$ & 6 \\
\hline & 15000 à $50000 \$$ & 8 \\
\hline & 50000 \$ et + & 6 \\
\hline \multirow[t]{3}{*}{ Scolarité : } & 14 ans et moins & 7 \\
\hline & 15 à 17 ans & 10 \\
\hline & Plus de 17 ans & 4 \\
\hline \multirow[t]{5}{*}{ Occupation : } & Sans emploi & 6 \\
\hline & Études & 1 \\
\hline & Retraite & 3 \\
\hline & Congé de maladie & 6 \\
\hline & Travail & 5 \\
\hline \multirow[t]{4}{*}{ Situation de vie : } & Célibataire & 6 \\
\hline & Séparé, divorcé & 5 \\
\hline & Avec conjoint & 9 \\
\hline & Avec autres & 1 \\
\hline \multirow[t]{3}{*}{ Âge : } & 20 à 30 ans & 4 \\
\hline & 31 à 50 ans & 6 \\
\hline & 50 ans et plus & 11 \\
\hline
\end{tabular}

\subsection{Acquisition des principes de base de la pleine conscience}

Entre le début (T1) et la fin de l'intervention (T2), il y a une différence significative du score global de pleine conscience $(\mathrm{t}=2,3 ; \mathrm{p}<, 04)$. Sur les deux 
dimensions qui composent le score global, soit moment présent et acceptation, cette différence s'observe uniquement sur la deuxième $(\mathrm{t}=3,3 ; \mathrm{p}<, 006)$. Trois mois après la fin du programme, ces différences ne sont plus présentes (voir tableau 3 ).

Tableau 3 - Impacts $d u$ programme sur les variables mesurées aux différents temps de mesure

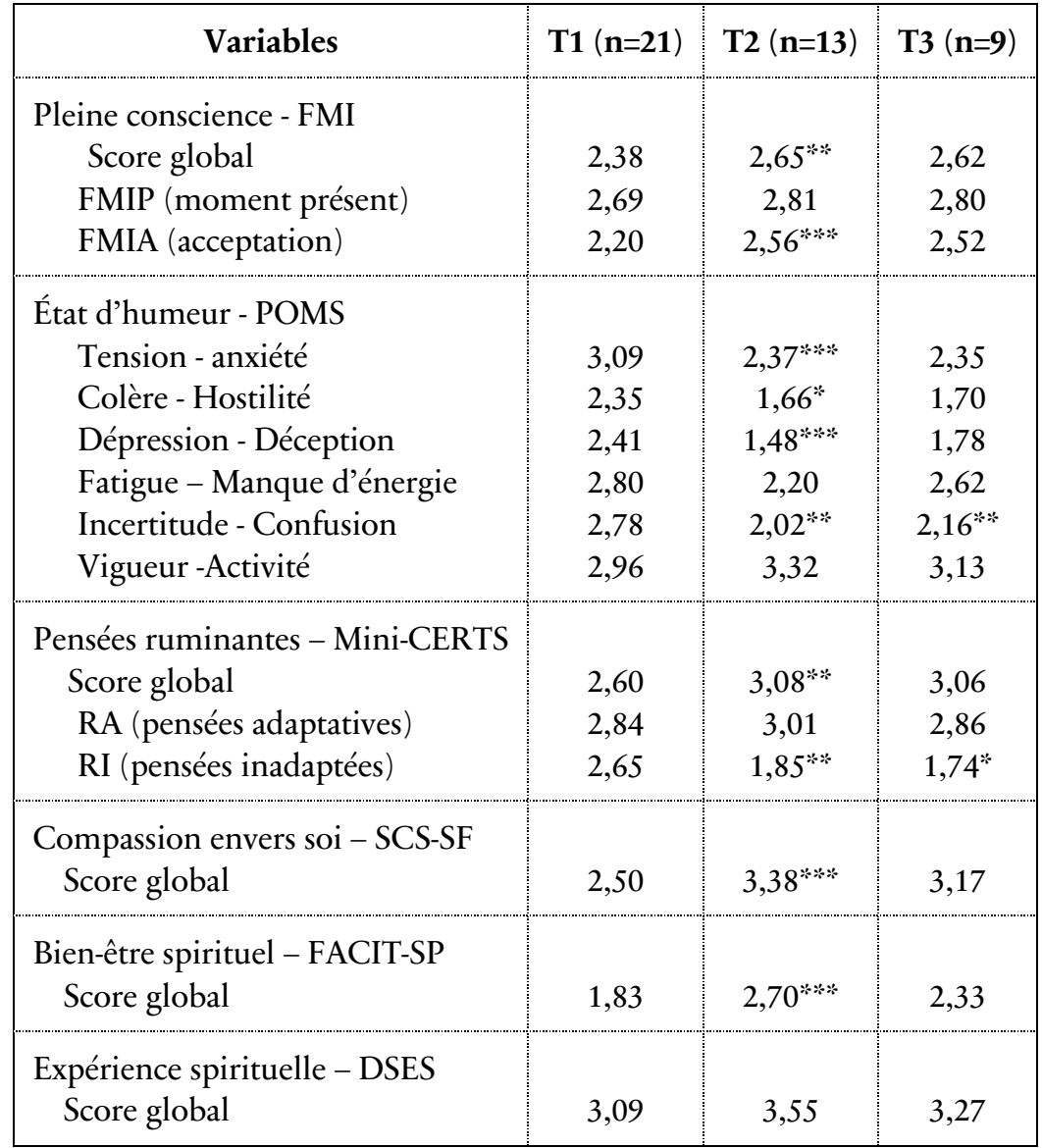

$$
* \mathrm{p}<0,1 ; * \mathrm{p}<0,05 ; * * \mathrm{p}<0,01
$$




\section{2 État de l'bumeur}

En regard de l'humeur, une différence significative s'observe entre le début et la fin de l'intervention aux sous-échelles Tension/Anxiété $(\mathrm{t}=3,31$; $\mathrm{p}<0,006$ ), Dépression/Déception ( $\mathrm{t}=3,98 ; \mathrm{p}<0,002)$ et Incertitude/Confusion $(\mathrm{t}=2,77 ; \mathrm{p}<0,017)$. Les analyses suggèrent que la sous-échelle Colère/Hostilité $(t=2,09 ; \mathrm{p}<0,059)$ tend également à diminuer. Après 3 mois, seule la diminution de la sous-échelle Incertitude/Confusion demeure significative $(t=2,40 ; \mathrm{p}<0,043)$.

\subsection{Pensées ruminantes adaptatives et inadaptées}

Lorsque l'on compare les réponses recueillies avant et immédiatement après le programme, il y a une différence significative sur le score global pour les pensées ruminantes $(t=2,64 ; p<0,022)$. Les pensées ruminantes inadaptées montrent une diminution ( $t=2,41 ; \mathrm{p}<0,033)$ alors qu'il n'y a pas de différence significative pour les pensées adaptées. Les analyses suggèrent que cette diminution tend à se maintenir $(\mathrm{t}=2,10 ; \mathrm{p}<0,069)$ trois mois après la fin du programme.

\subsection{Compassion envers soi}

Les analyses montrent une augmentation significative de compassion des participants envers eux-mêmes entre le début et la fin de l'intervention $(t=4,20 ; p<0,001)$. Toutefois, cette augmentation n'est plus notable trois mois plus tard.

\subsection{Bien-être spirituel}

Les analyses montrent qu'au T2 les participants ont une augmentation significative de leur bien-être spirituel $(t=4,20 ; p<0,001)$. Toutefois, cette augmentation ne demeure pas significative dans le temps.

\subsection{Expérience spirituelle}

Lorsque l'on compare les réponses recueillies avant et après le programme, l'expérience spirituelle ne montre aucune différence significative. 


\section{Discussion}

Les interventions en soins spirituels au CSsanté proposent des activités visant à soutenir les patients au cours d'une période généralement difficile dans leur vie. Comme l'intention du programme de méditation MBSR est de réduire la souffrance, d'augmenter les émotions positives et d'améliorer la qualité de vie (Vago et Silbersweig 2012), il s'inscrit tout naturellement en cohérence avec la visée globale de nos activités. Le protocole de recherche proposait l'évaluation des retombées d'un programme de MPC semblable à celui inauguré par Kabat-Zinn, mais légèrement modifié puisqu'il offrait aux participants la possibilité de s'exprimer sur leurs propres croyances. Nous cherchions à évaluer les répercussions de ce programme modifié sur les dimensions psychique et spirituelle des participants.

Les résultats de cette recherche suggèrent que les participants ont, à la fin du programme, acquis certains principes de base de la pratique de la méditation, notamment en démontrant une plus grande ouverture face aux expériences négatives. Selon nos résultats, les ateliers que nous avons proposés aident à réduire l'anxiété (Tension/Anxiété dans le POMS), l'humeur dépressive (Dépression/Déception) et la confusion (Incertitude/Confusion), ainsi que les pensées ruminantes, particulièrement celles inadaptées. L'étude suggère également que nos ateliers favorisent la compassion envers soi et le bien-être spirituel.

Pour la plupart de ces variables, les résultats généraux sont en cohérence avec ceux des recherches consultées. Le fait qu'une thérapie de pleine conscience apporte des bienfaits aux participants immédiatement après la fin du programme a été rapporté, notamment en ce qui a trait à l'anxiété (Chan et Larson 2015 ; Khoury et al. 2013 ; Zhang et al. 2015) et la dépression (Jain et al. 2015 ; Strauss et al. 2014). L'effet positif sur les pensées ruminantes avait aussi été établi, notamment dans l'étude de Heeren et Philippot (2011). Selon Gu et al. (2015), le développement des habiletés de pleine conscience contribue à être moins réactif face à ses propres expériences, ce qui peut expliquer les effets positifs au plan psychique.

Notre étude visait également la vérification de l'effet à long terme du programme. Trois mois après la fin de l'expérimentation, nous avons noté peu d'amélioration significative en regard du Temps 1. L'absence de 
suivi formel entre les Temps 2 et 3, ainsi que le nombre peu élevé de participants lors du Temps 3 nous empêchent de tirer des conclusions en ce qui a trait à l'effet du programme à long terme. Ces résultats non significatifs sont donc difficilement interprétables quoiqu'ils puissent indiquer une tendance vers des effets bénéfiques et nous renseigner sur l'importance d'un suivi à la suite d'un programme de méditation. Si aucune proposition de pratiques ne soutient le participant, il peut être difficile d'observer le maintien à long terme des bienfaits de la méditation (Grow et al. 2014 ; Marchand 2013).

\subsection{Mesure des répercussions sur la spiritualité}

Deux instruments de mesure spécifiques avaient été retenus afin d'évaluer l'impact du programme sur la spiritualité des participants, soit le FACITSP et le DSES. Le premier (FACIT-SP), plus séculier par nature (Carmody et al.2008), facilite l'évaluation dans un contexte comme le nôtre. Au temps 2 , les résultats indiquent une augmentation des scores, révélant une amélioration du bien-être spirituel, tel que mesuré par le FACIT-SP. Tout comme pour les autres variables, cet effet ne semble pas se maintenir à plus long terme puisqu'il devient non-significatif trois mois après la fin du programme. Cependant, bien que non statistiquement significative, on remarque tout de même une tendance du maintien de cette amélioration. L'étude de Würtzen et al. (2015), qui avait aussi mesuré le bien-être spirituel en utilisant le FACIT-SP, indique également une diminution de l'effet à plus long terme.

Le deuxième, le DSES, mesure spécifiquement les effets du programme sur l'expérience spirituelle ${ }^{4}$. Les résultats des analyses ne révèlent pas de différence entre les temps de mesure, ce qui indiquerait que le programme de méditation n'aurait eu aucun effet sur l'expérience spirituelle des participants, telle que mesurée par le DSES. Cependant, plusieurs questions dans le libellé faisaient directement référence à Dieu, ce qui peut avoir suscité chez les participants une association avec la religion, qui en

4 Le terme expérience spirituelle est abordé dans ce texte en lien avec les résultats obtenus suite à l'application de l'outil DSES. 
contexte québécois continue de susciter de la méfiance et même des préjugés.

Les résultats ne nous permettent pas d'affirmer que les éléments nouveaux introduits en regard du programme de base développé par KabatZinn aient engendré des impacts positifs ou négatifs sur les participants. Les données observées immédiatement après les huit (8) semaines sont semblables aux résultats constatés dans d'autres études, particulièrement dans des méta-analyses (Chan et Larson 2015 ; Khoury et al. 2013 ; Zhang et al. 2015 ; Jain et al. 2015 ; Strauss et al. 2014 ; Gu et al. 2015). Toujours sur la base de nos résultats, nous constatons des effets modérément positifs sur cinq des six variables investiguées. Ceci suggère que le programme, malgré la modification de certains éléments mineurs, ne semble pas changer les conclusions généralement observées au sein de la littérature. D'autres recherches devraient cependant être conduites afin d'évaluer l'effet d'ajouts d'éléments en lien avec la Transcendance sur un programme de MPC. Par exemple, compte tenu du contexte québécois, qui est fortement laïcisé, il serait souhaitable de comparer deux programmes distincts : le MBSR dans sa forme originale strictement séculière et un autre programme de méditation de pleine conscience où les participants ont la possibilité d'y intégrer leur propre expérience avec Dieu ou avec un Transcendant.

Les travaux de Carmody et al. (2008) ont démontré que le programme MBSR - sans l'ajout, comme nous l'avons fait, d'éléments en lien avec un Transcendant - peut produire des impacts positifs sur le bien-être spirituel, tel que mesuré par le FACIT-SP. Cependant, les questions concernant les répercussions sur la spiritualité des participants restent nombreuses. Est-il possible d'affirmer que l'amélioration de certains indicateurs psychologiques (baisse de l'humeur dépressive, de l'anxiété, de la confusion, etc.) favorise le bien-être spirituel ou si c'est uniquement le programme qui engendre ce bien-être? À l'inverse, est-ce l'amélioration du bien-être spirituel qui favorise une meilleure santé psychique ? Sur la base de leur étude, Greeson et al. (2011) soutiennent que les effets bénéfiques de la MPC sur la santé mentale et le bien-être de la personne s'expliquent par deux facteurs combinés : une augmentation de la présence attentive et l'expérience d'un lien avec un Transcendant (mesurée avec le DSES). Autrement dit, la combinaison de ces deux facteurs expliquerait l'efficacité du programme MBSR. Toujours selon Greeson et al., « l'augmentation de la spiritualité, caractérisée par la conscience d'un lien avec un Transcendant 
dans la vie quotidienne, n'est pas seulement un résultat important de la formation à la MPC, mais également un mécanisme clé par lequel le MBSR favorise la santé mentale » (2011, 8, nous traduisons).

Ces questions sont majeures spécialement pour les professionnels des soins spirituels. Le caractère imprécis et difficilement opérationnalisable de ce qu'on entend par "spiritualité » soulève de nombreuses questions et touche la présente recherche. Lorsque Greeson et al. évoquent «l'accroissement dans la vie quotidienne de la conscience d'un lien avec un Transcendant» $(2011,8)$, il demeure difficile d'évaluer de quelle manière cette augmentation peut être mesurée en faisant référence à des concepts autres que religieux. En effet, certaines des questions proposées par le DSES et le FACIT-SP supposent un lien avec Dieu ou avec des croyances. Le FACIT-SP, pourtant considéré plus séculier que le DSES, demeure un outil imprécis pour mesurer la vie spirituelle d'une personne qui ne se réfere pas à des croyances objectivées. En effet, neuf des douze questions du FACIT-SP ne supposent pas de liens directs avec la foi et les croyances et pourraient donc être, selon l'angle envisagé, confondues avec la dimension psychique. Les trois questions qui envisagent la spiritualité en référence avec la foi et les croyances pourraient, elles, être confondues avec la dimension religieuse. Toute mesure de la dimension spirituelle demeure donc complexe et surtout discutable selon les contextes d'intervention. La spiritualité réfere-t-elle essentiellement à des notions liées à une relation avec un Transcendant ou à des croyances? Si oui, cela apparaît réducteur pour de nombreuses personnes, notamment au Québec, qui ne font pas appel, dans l'expression de leur vie spirituelle, à un Dieu ou à un Transcendant ou même à des croyances. Afin de contourner ce problème, Greeson et al. (2011 et 2015) ont d'ailleurs retiré la mention de Dieu dans les items du DSES et l'ont remplacée par la notion d'une force supérieure. La question demeure complexe et, en tant que professionnels des soins spirituels, il est essentiel de prendre en compte, dans l'évaluation de nos programmes, les difficultés liées aux outils qui mesurent les conséquences de nos interventions, notamment celles reliées aux effets de la méditation.

\subsection{Limites de l'étude}

L'absence de résultats significatifs 3 mois après la fin du programme (T3) peut être expliquée par le nombre limité de participants : n'ayant pas la 
puissance statistique nécessaire, il est impossible de déterminer si les différences observées sont statistiquement significatives. De plus, nous devons prendre en compte le haut taux de désistement des participants. Le faible nombre de participants indique les difficultés de recrutement dans un contexte marqué par la fin d'une période d'hospitalisation. Nous sommes contraints par les conditions de santé des patients qui peuvent faire en sorte qu'il devient impossible pour un participant de poursuivre sa démarche. Mais ceci pourrait aussi indiquer un désintérêt à l'endroit du programme. Il est impossible de déterminer quelles sont les raisons expliquant le désistement des participants dans le cadre de la présente recherche. Une rencontre ultérieure aurait pu apporter des éléments de réponse à cette question. Ainsi, le fait que toutes les personnes n'aient pas complété le T2 et le T3 limite la force des conclusions de l'étude. Sans les données de ces personnes, il est impossible de déterminer si le programme a amélioré leur situation. Il est donc impossible de conclure de manière définitive si le programme de méditation que nous avons évalué dans la présente étude fonctionne pour tout le monde ou seulement pour les personnes ayant des caractéristiques spécifiques qui favorisent la participation à terme au programme (Smith et al. 2015).

L'ajout d'un volet qualitatif (Cairns et Murray 2015; Mitchell et Heads 2015) aurait été pertinent, car il aurait permis de mieux comprendre le vécu des participants. Cela aurait été d'autant plus avisé que le programme utilisé comportait une ouverture à la discussion sur la spiritualité, ce qui est peu fréquent dans les diverses adaptations du programme de MBSR.

L'absence d'un groupe contrôle randomisé ou d'un groupe de comparaison est un autre aspect qui limite la portée de l'étude, car il est impossible de confirmer que c'est bien la participation au programme qui a induit les changements observés entre le T1 et le T2 (Davidson et Kaszniak 2015 ; Greeson et al. 2015 ; Smith et al. 2015).

\section{Conclusion}

L'originalité du présent projet a été de construire un programme qui croisait des éléments de la méditation pleine conscience à des éléments d'ouverture à un Transcendant ou à un Dieu. Les résultats obtenus à la suite de la participation au programme de méditation Soin spirituel pour un mieux- 
être concordent globalement avec l'ensemble des recherches consultées, à savoir des effets modérément positifs, si on exclut l'extension dans le temps (après trois mois). D'autres recherches seraient nécessaires afin de mieux comprendre comment le rôle de la spiritualité a une incidence sur l'efficacité du programmes.

\section{Références}

BAer, R. A. (2003), « Mindfulness Training as a Clinical Intervention. A Conceptual and Empirical Review », Clinical Psychology. Science and Practice, 10, p. 125-143.

5 Notice de présentation des auteurs et autrices :

Bruno Bélanger (baccalauréat en théologie, Université Laval, 1980 ; baccalauréat, 1991 et M.A. 1994 en sciences politiques, Université de Montréal) est l'auteur principal de : (2020), "The Quebec Model of Recording Spiritual Care. Concepts and Guidelines », dans S. Peng-Keller et D. NeuHold, dir., Charting Spiritual Care. The Emerging Role of Chaplaincy Records in Global Health Care, Cham, Switzerland, Springer, p. 53-78.

https://link.springer.com/chapter/10.1007/978-3-030-47070-8_4

Cécile Charbonneau (Ph.D. en psychologie, Université Laval, 1995) s'intéresse particulièrement aux soins spirituels et aux soins palliatifs. Elle a publié, entre autres: (2013), "Identification of Spiritual and Religious Needs in Terminally Ill Patients Receiving Palliative Home-care ", Journal for the Study of Spirituality, 3, p. 33-45.

Line Beauregard (M.A. en psychologie, 1990 ; et Ph.D. en service social, 2002, Université Laval) est chercheure au Centre Spiritualitésanté de la Capitale Nationale ; elle a publié : (avec B. Bélanger, 2018), « Recherche sur la spiritualité. Les défis du recrutement », dans A. MARIN, B. EYSERMANN et M. T. GIROUX, dir., Recrutement et consentement à la recherche. Réalités et défis éthiques, Sherbrooke, Éditions de l'Université de Sherbrooke, p. 106-114, https ://doi.org/10.17118/11143/14115.

Christiane Biron (baccalauréat ès arts, majeure en théologie et mineure en psychologie, Université Laval, 1993 ; M.A. en accompagnement spirituel en milieu de santé, Université Laval, 2004) est intervenante en soins spirituels depuis plus de 20 ans. Elle est l'instigatrice et l'animatrice du programme Soin spirituel au mieux-être. A publié sur ce sujet : (2014), «Et si la porte de sortie se trouvait à l'intérieur... », SpiritualitéSanté, 7, p. 30-33. Stéphane Poulin (M.A. en psychologie, Université Laval, 2000 ; études de médecine, Université de Montréal, 2002, et de psychiatrie, Université Laval, 2007 ; fellowship en neurologie comportementale et neuropsychiatrie, McLean Hospital et Massachusetts General Hospital, 2009) a publié entre autres : (2017), «Alzheimer's Disease Neuroimaging Initiative. Risk Factors, Neuroanatomical Correlates, and Outcome of Neuropsychiatric Symptoms in Alzheimer's Disease ", Journal of Alzheimers Disease, 60, p. 483-493. 
(2010), "Mindfulness - and Acceptance-Based Interventions and Processes of Change », dans R. A. BAER, Assessing Mindfulness and Acceptance Processes in Clients. Illuminating the Theory and Practice of Change, Oakland, CA, New Harbinger Publications, p. 1-21.

Barnhofer, T., C. Crane, E. Hargus, M. Amarasinghe, R. Winder et J.M.G. WiLliams (2009), « Mindfulness-Based Cognitive Therapy as a Treatment for Chronic Depression. A Preliminary Study », Behaviour Research and Therapy, 47, p. 366-373.

Bawa, F. L., S. W. Mercer, R. J. Atherton, F. Clague, A. Keen, N. W. SCOTT et C. M. Bond (2015), «Does Mindfulness Improve Outcomes in Patients with Chronic Pain? Systematic Review and MetaAnalysis », The British Journal of General Practice. The Journal of the Royal College of General Practitioners, 65 ( $\mathrm{n}^{\circ}$ 635), p. 387-400.

BIRON, C. (2014), «Et si la porte de sortie se trouvait à l'intérieur... », Spiritualitésanté, 7/3, p. 30-33.

Brown, K. W., R. M. Ryan et J. D. Creswell (2007), « Mindfulness. Theoretical Foundations and Evidence for its Salutary Effects ", Psychological Inquiry, 18, p. 211-237.

Cairns, V. et C. Murray (2015), « How do the Features of MindfulnessBased Cognitive Therapy Contribute to Positive Therapeutic Change ? A Meta-Synthesis of Qualitative Studies », Behavioural Cognitive Psychotherapy, 43, p. 342-359.

Carlson, L. E., Z. Ursuliak, E. Goodey, M. Augen et M. Speca (2001), "The Effects of a Mindfulness Meditation-Base Stress Reduction Program on Mood and Symptoms on Stress in Cancer Outpatient. 6-Month Follow-up », Support Care Cancer, 9, p. 112-123.

Carmody, J., G. Reed, J. Kristeller et P. Merriam (2008), « Mindfulness, Spirituality, and Health-Related Symptoms », Journal of Psychosomatic Research, 64, p. 393-403.

CHAN, R. R. et J. L. LARSON (2015), «Meditation Interventions for Chronic Disease Populations. A Systematic Review », Journal of Holistic Nursing, 33, p. 351-365. 
Chen, K. W., C. C. Berger, E. Manheimer, D. Forde, J. Magidson, L. DaChMAn et C. W. LejueZ (2012), « Meditative Therapies for Reducing Anxiety. A Systematic Review and Meta-Analysis of Randomized Controlled Trials », Depress Anxiety, 29, p. 545-562.

Creswell, J. D. et E. K. Lindsay (2014), « How Does Mindfulness Training Affect Health? A Mindfulness Stress Buffering Account », Current Directions in Psychological Science, 23, p. 401-407.

Davidson, R. J. et A. W. KaszniaK (2015), « Conceptual and Methodological Issues in Research on Mindfulness and Meditation », The American Psychologist, 70, p. 581-592.

Douilliez, C. et P. PHilippot (2012), «Pensées répétitives constructives et non constructives chez les perfectionnistes inadaptés ", Journal de la Thérapie Comportementale et Cognitive, 22/2, p. 68-74.

Evans, S., S. Ferrando, M. Findler, C. Stowell, C. SMart et D. Haglin (2008), " Mindfulness-based Cognitive Therapy for Generalized Anxiety Disorder », Journal of Anxiety Disorders, 22, p. 716-721.

Fillion, L. et P. GAGNON (1999), «French Adaptation of the Shortened Version of the Profile of Mood States ", Psychological Reports, 84, p. 188-190.

Fitchett, G. et A. L. CANADA (2010), « The Role of Religion/Spirituality in Coping with Cancer. Evidence, Assessment, and Intervention », dans J. C. Holland, dir., Psycho-Oncology, New York, Oxford University Press, p. 440-446.

Fournier, C. (2014), «La méditation, un sujet d'actualité », Spiritualitésanté, $7 / 3$, p. 20-25.

Gonçalves, J., G. Lucchetti, P. Menezes et H. Vallada (2015), « Religious and Spiritual Interventions in Mental Health Care. A Systematic Review and Meta-Analysis of Randomized Controlled Clinical Trials », Psychological Medicine, 45, p. 2937-2949.

GrabovaC, A. (2014), «The Stages of Insight. Clinical Relevance for Mindfulness-Based Interventions », Mindfulness, 6, p. 589-600.

Greeson, J. M., D. M. WebBer, M. J. SMOSKI, J. G. BRANTLEY et al. (2011), "Changes in Spirituality Partly Explain Health-Related Quality of 
Life Outcomes after Mindfulness-Based Stress Reduction », Journal of Behavioral Medecine, 34, p. 508-518.

Greeson, J. M., M. J. Smoski, E. C. Suarez, J. G. Brantley, A. G. Ekblad, T. R. LYNCH et R. Q. WOlever (2015), « Decreased Symptoms of Depression after Mindfulness-Based Stress Reduction. Potential Moderating Effects of Religiosity, Spirituality, Trait Mindfulness, Sex, and Age ", Journal of Alternative and Complementary Medecine, 21, p. 166-174.

Grow, J. C., S. E. Collins, E. N. Harrop et G. A. Marlatt (2014), « Enactment of Home Practice Following Mindfulness-Based Relapse Prevention and its Association with Substance-Use Outcomes », Addictive behaviors, 40, p. 16-20.

Gu, J., C. Strauss, R. Bond et K. Cavanagh (2015), « How do Mindfulness-Based Cognitive Therapy and Mindfulness-Based Stress Reduction improve Mental Health and Wellbeing? A Systematic Review and Meta-Analysis of Mediation Studies », Clinical Psychology Review, 37, p. 1-12.

Heeren, A. et P. Philippot (2011), "Changes in Ruminative Thinking mediate the Clinical Benefits of Mindfulness. Preliminary Findings ", Mindfulness, 2, p. 8-13.

Hofmann, S. G., A. T. SAwYer, A. A. WitT et D. OH (2010), « The Effect of Mindfulness-Based Therapy on Anxiety and Depression. A MetaAnalytic Review », Journal of Consulting and Clinical Psychology, 78, p. $169-183$.

Jain, F. A, R. N. Walsh, S. J. Eisendrath, S. Christensen et B. R. CAhN (2015), «Critical Analysis of the Efficacy of Meditation Therapies for Acute and Subacute Phase Treatment of Depressive Disorders. A Systematic Review », Psychosomatics, 56, p. 140-152.

Kabat-ZINN, J. (1982), « An Outpatient Program in Behavioural Medicine for Chronic Pain Patients Based on the Practice of Mindfulness Meditation. Theoretical Considerations and Preliminary Results », General Hospital Psychiatry, 4, p. 33-47.

(2003), « Mindfulness-Based Intervention in Context. Past, Present, Future. Clinical Psychology », Science and Practice, 10, p. 144-156. 
Koenig, H. K. (2004), «Religion, Spirituality, and Medecine. Research Findings and Implications for Clinical Practice », Southern Medical Journal, 97, p. 1194-1200. (2012), « Religion, Spirituality, and Health. The Research and Clinical Implications », INRS Psychiatry, vol. 2012, art. ID 278730, non paginé, https ://doi.org/10.5402/2012/278730

Khoury, B., T. LeComte, G. Fortin et al. (2013), « Mindfulness-Based Therapy. A Comprehensive Meta-Analysis », Clinical Psychology Review, 33, p. 763-771.

MCNaIR, D. M., M. LORR et L. F. Droppleman (1971), Manual for the Profile of Mood States, San Diego, CA, Educational and Industrial Testing Services.

MarChand, W.R. (2013), « Mindfulness Meditation Practices as Adjunctive Treatments for Psychiatric Disorders ", Psychiatric Clinics of North America, 36, p. 141-152.

Mitchell, M. et G. Heads (2015), « Staying Well. A Follow Up of a 5Week Mindfulness Based Stress Reduction Programme for a Range of Psychological Issues », Community Mental Health Journal, 51, p. 897-902.

Murray, S. A., M. Kendall, K. Boyd, A. Worth et T. F. Benton (2004), « Exploring the Spiritual Needs of People dying of Lung Cancer or Heart Failure. A Prospective Qualitative Interview Study of Patients and their Carers », Palliative Medicine, 18, p. 39-45.

NefF, K. D. (2003), « The Development and Validation of a Scale to measure Self-Compassion », Self and Identity, 2, p. 223-250.

ОкоN, T.R. (2005), « Spiritual, Religious and Existential Aspects of Palliative Care », Journal of Palliative Medicine, 8, p. 392-413.

Peterman, A. H., G. Fitchett, M. J. Brady, L. Hernandez et D. Cella (2002), «Measuring Spiritual Well-Being in People with Cancer. The Functional Assessment of Chronic Illness Therapy-Spiritual Well-Being Scale (FACIT-Sp) », Annals of Behavioral Medicine, 24, p. 49-58. 
PuCHALSKI, C. M. (2007), « Spirituality and the Care of Patients at the Endof-Life. An Essential Component of Care », Omega, 56, p. 33-46.

Shacham, S. (1983), "A Shortened Version of the Profile of Mood States », Journal of Personality Assessment, 47, p. 305-306.

Shapiro, S. L., L. E. Carlson, J. A. Astin et B. Freedman (2006), « Mechanisms of Mindfulness », Journal of Clinical Psychology, 6, p. 373-386.

Sinclair, S., J. Pereira et S. RAfFin (2006), «A Thematic Review of the Spiritual Literature within Palliative Care», Journal of Palliative Medicine, 9, p. 464-479.

Smith, B., K. Metzker, R. Waite et P. Gerrity (2015), «Short-Form Mindfulness-Based Stress Reduction reduces Anxiety and improves Health-Related Quality of Life in an Inner-City Population », Holistic Nursing Practice, 29, p. 70-77.

Speca, M., L. E. Carlson, E. Goodey et M. Angen (2000), «A Randomized, Wait-List Controlled Clinical Trial. The Effect of a Mindfulness Meditation-Based Stress Reduction Program on Mood and Symptoms of Stress in Cancer Outpatients », Psychosomatic Medicine, 62, p. 613-622.

Strauss, C., K. Cavanagh, A. Oliver et D. Pettman (2014), « Mindfulness-Based Interventions for People Diagnosed with a Current Episode of an Anxiety or Depressive Disorder. A Meta-Analysis of Randomised Controlled Trials », PloS one, 9/4, non paginé,

\section{https ://doi.org/10.1371/journal.pone.0096110}

Trousselard, M., D. Steiler, C. Raphel, C. Cian, R. Duymedjian, D. ClaveriE et F. CANINI (2010), «Validation of a French Version of the Freiburg Mindfulness Inventory-Short Version. Relationships between Mindfulness and Stress in Adult Population », BioPsychoSocial Medicine, 4, non paginé, https ://doi.org/10.1186/1751-0759-4-8.

UNDERWOOD, L. G. (2006), « Ordinary Spiritual Experience. Qualitative Research, Interpretive Guidelines, and Population Distribution for the Daily Spiritual Experience Scale ", Archive for the Psychology of Religion, 26/1, p. 181-218. 
(2019), « Using the Daily Spiritual Experience Scale in Research and Practice », (Preprint) Applelane Press, http://www.dsescale.org/

Vago, D. R. et D. A. Silbersweig (2012), « Self-Awareness, Self-Regulation, and Self-Transcendence (S-ART). A Framework for Understanding the Neurobiological Mechanisms of Mindfulness ", Frontiers in Human Neuroscience, 6, p. 1-30.

Visser, A., B. Garssen et A. Vingerhoets (2010), « Spirituality and WellBeing in Cancer Patients. A Review », Psychooncology, 19, p. 565-572.

Walach, H., N. BuChHeld, V. ButTenmüler, N. KleinkneCht et S. SCHMIDT (2006), « Measuring Mindfulness - the Freiburg Mindfulness Inventory », Personality and individual differences, 40, p. 15431555 .

Williams, M., J. Teasdale, Z. Segal et J. Kabat-Zinn (2009), Méditer pour ne plus déprimer, Paris, Odile Jacob.

Würtzen, H., S. O. Dalton, J. Christensen, K. K. Andersen, P. Elsass, H. L. FLYGER et al. (2015), » Effect of Mindfulness-Based Stress Reduction on Somatic Symptoms, Distress, Mindfulness and Spiritual Wellbeing in Women with Breast Cancer. Results of a Randomized Controlled Trial », Acta Oncologica, 54, p. 712-719.

Zhang, M. F., Y. S. Wen, W. Y. LiU, L. F. Peng, X. D. Wu et Q. W. LiU (2015), « Effectiveness of Mindfulness-Based Therapy for Reducing Anxiety and Depression in Patients With Cancer. A Meta-Analysis », Medicine, 94, non paginé, doi: 10.1097/MD.0000000000000897

\section{Résumé}

Au cours des dernières années, dans la foulée du programme de méditation pleine conscience élaboré par Jon Kabat Zinn, les services de soins spirituels intègrent de plus en plus la médiation au sein de leurs interventions. Cet article présente les résultats d'une recherche visant à évaluer les effets d'un programme de méditation « Soin spirituel pour un mieux-être » sur diverses dimensions psychiques et spirituelles. Les données ont été recueillies auprès de 21 patients suivis en externe en psychiatrie à l'Hôpital de l'Enfant-Jésus selon 3 temps de mesure : avant le programme, immédiatement à la fin du 
programme et 3 mois après. Les résultats suggèrent une amélioration chez les patients immédiatement à la fin du programme mais qui tend à s'atténuer 3 mois plus tard. Cette recherche pose, entre autres, des questions concernant les mesures d'évaluation de la dimension spirituelle.

\section{Abstract}

In recent years, in the wake of Jon Kabat Zinn's mindfulness meditation program, spiritual care services are increasingly incorporating meditation into their intervention. This article presents the results of a research to evaluate the effects of a "Spiritual Care for a Well-being" meditation program on various psychic and spiritual dimensions. Data were collected from 21 outpatients in psychiatric care at the Enfant-Jésus Hospital measured at 3 time-intervals: before the program, immediately at the end of the program and 3 months thereafter. The results suggest an improvement in patients immediately at the end of the program, which however tends to subside 3 months later. This research raises, among other things, questions concerning measurement of the spiritual dimension. 